\title{
Sepsis-induced heparin resistance during extracorporeal membrane oxygenation
}

\author{
Ali Hage MD, Martha Louzada MD, Bob Kiaii MD
}

Cite as: CMAJ 2019 March 11;191:E283-5. doi: 10.1503/cmaj.181061

A 41-year-old woman was transferred from a peripheral hospital to the intensive care unit (ICU) at our hospital with a diagnosis of pneumosepsis and acute respiratory distress syndrome secondary to Legionella pneumonia (postadmission day 0 ). The patient had a history of gastroesophageal reflux disease, allergic rhinitis and migraine, but was otherwise healthy. Her weight was $90.9 \mathrm{~kg}$. She had not been previously exposed to heparin.

In the ICU, the patient was intubated. Her oxygenation and ventilation rapidly deteriorated and she developed severe hypercapnia and hypoxemia. Because various modalities of ventilation, including putting the patient in a prone position, were not successful, we made the decision to proceed with veno-venous extracorporeal membrane oxygenation (ECMO) through the right internal jugular vein and the right femoral vein on postadmission day 4 .

On that day, the baseline activated partial thromboplastin time (aPTT) before initiation of unfractionated heparin was 29 (reference range 23-32) s. Complete blood count before the initiation of ECMO showed leukocytosis (white blood cells 17.7 [reference range 4.0-10.0] $\times 10^{9} / \mathrm{L}$ ), anemia (hemoglobin 96 [reference range 115-160] g/L) and thrombocytopenia (platelets 68 [reference range $150-400] \times 10^{9} / \mathrm{L}$ ). We began veno-venous ECMO, using an unfractionated heparin-coated circuit, on postadmission day 4 at 0315 after the administration of a 30000 -unit bolus of unfractionated heparin.

At 1140 on the same day, the aPTT remained subtherapeutic at a maximal value of $29 \mathrm{~s}$. The international normalized ratio (INR) was 1.1 (reference range 0.9-1.1). We started a heparin infusion of 5 units $/ \mathrm{kg} / \mathrm{hr}$ at 1229 on postadmission day 4, and subsequently increased it to $9.6 \mathrm{units} / \mathrm{kg} / \mathrm{hr}$ at 1019 on postadmission day 5, then to 12 units $/ \mathrm{kg} / \mathrm{hr}$ later that day, at 1533. In addition, we administered 5 supplemental boluses of unfractionated heparin (on postadmission day 5: 4000 units at 0715, 10000 units at 1055, 5000 units at 1706, 3000 units at 1755; on postadmission day 6: 4000 units at 0010). We also administered 4 units of fresh frozen plasma at 1711 on postadmission day 5 (aPTT $26 \mathrm{~s}$ and INR 1.1 at 1600 on postadmission day 5 before administration of fresh frozen plasma, and aPTT $29 \mathrm{~s}$ and INR 1.1 at 2300 on that day, 6 hours after administration of fresh frozen plasma).

\section{KEY POINTS}

- Correctly diagnosing heparin resistance in patients on extracorporeal membrane oxygenation (ECMO) is challenging.

- In patients with sepsis, the differential diagnosis for heparin resistance is large, and includes acquired antithrombin deficiency and non-antithrombin-mediated sequestration of heparin.

- When thrombocytopenia occurs in patients with sepsis who are on ECMO, heparin-induced thrombocytopenia and disseminated intravascular coagulation must be ruled out before sepsis can be presumed to be the cause.

- Direct thrombin inhibitors can be used as anticoagulation in acquired heparin resistance with ECMO, and the choice of this type of circuit (heparin-coated v. non-heparin-coated) should take into consideration the result of the heparin-induced thrombocytopenia assay.

At 2300 on postadmission day 5, the patient's aPTT remained subtherapeutic at $29 \mathrm{~s}$, despite the continuous infusion and the multiple boluses of unfractionated heparin. Additionally, the patient's hemoglobin dropped to $65 \mathrm{~g} / \mathrm{L}$ despite the recent transfusion of 3 units of packed red blood cells, and her platelets dropped to $18 \times 10^{9} / \mathrm{L}$. Hemolysis workup was negative and the patient's INR and aPTT remained subtherapeutic at 1.1 and $29 \mathrm{~s}$, respectively. The patient's fibrinogen level was normal at 2.24 (reference range $2.00-4.00) \mathrm{g} / \mathrm{L}$, but her antithrombin level was low, at 0.63 (reference range $0.83-1.28$ ) $\mathrm{U} / \mathrm{mL}$. The patient had preserved liver function (alanine transaminase: 16 [reference range $\leq 33$ ] $\mathrm{U} / \mathrm{L}$; aspartate transaminase: 38 [reference range $\leq 32] \mathrm{U} / \mathrm{L}$ ) and no evidence of proteinuria (protein in urine: $0 \mathrm{~g} / \mathrm{L}$ ). After this, we administered 1000 IU of antithrombin III concentrate along with 2 units of platelets at 2300 on postadmission day 5. However, 4 hours later (at 0305 on postadmission day 6), the patient's aPTT remained subtherapeutic at $33 \mathrm{~s}$ and her platelets were $37 \times 10^{9} / \mathrm{L}$.

At this point, the medical team was concerned about the viability of the veno-venous ECMO, owing to the risk of circuit thrombosis, which would put the safety of the patient at risk. The probability of a diagnosis of heparin resistance was made on the basis of our inability to reach a therapeutic aPTT after giving 61000 units of unfractionated heparin in less than 24 hours, 
more than the minimum needed value of 35000 daily units established by the currently available literature. ${ }^{1}$ We decided to stop the heparin and begin argatroban infusion at a rate of $1 \mu \mathrm{g} / \mathrm{kg} / \mathrm{min}$ with a target aPTT of $45-90 \mathrm{~s}$. We began argatroban at 0357 on postadmission day 6 . About 2 hours later, the patient's aPTT increased to $63 \mathrm{~s}$ and then to $74 \mathrm{~s}$. Her platelets remained low, at $25 \times 10^{9} / \mathrm{L}$.

Heparin-induced thrombocytopenia enzyme-linked immunosorbent assay (ELISA) assay was performed twice; it came back negative both times. We monitored the argatroban infusion rate via aPTT levels and decreased the dose of infusion to $0.5 \mu \mathrm{g} / \mathrm{kg} / \mathrm{min}$, given that the patient's aPTT levels remained therapeutic at this lower dose of argatroban.

Five days after the initiation of the argatroban infusion (postadmission day 11), the patient's aPTT levels remained therapeutic at $62 \mathrm{~s}$ and her platelet count increased gradually to reach $174 \times 10^{9} / \mathrm{L}$. The veno-venous ECMO was functioning well under argatroban without any sign of filter dysfunction or clot formation. Eight days later, the patient's respiratory status had improved and we could wean her off the veno-venous ECMO. Twenty-five days after admission to hospital, the patient had completely recovered and was discharged home. We saw her at the hospital 7 months after her discharge and she was doing well.

\section{Discussion}

This is a case of a severe presentation of heparin resistance in a patient on veno-venous ECMO requiring optimal anticoagulation to prevent clot formation in the circuit and embolic thrombosis. Despite the administration of several boluses of unfractionated heparin, the patient's aPTT remained subtherapeutic. We were confidently able to rule out other causes of thrombocytopenia, namely heparin-induced thrombocytopenia and disseminated intravascular coagulation.

\section{Causes of heparin resistance}

Our main hypothesis was that, although rare, the heparin resistance could be secondary to an acquired antithrombin deficiency in the setting of severe sepsis, ${ }^{2}$ as depicted by the low level of antithrombin in our patient. The reduction in antithrombin levels in the setting of sepsis could be secondary to a downregulation in the production of antithrombin, which is aggravated by an increased turnover of plasmatic proteins. ${ }^{2}$ Moreover, low antithrombin levels are also found in the presence of heparin. ${ }^{3}$ In addition, both liver failure and proteinuria could lead to low antithrombin III levels, but this was not the case for our patient, as she had preserved liver function and no evidence of proteinuria. Therefore, we administered antithrombin III concentrate to replete the patient's low level of antithrombin, and also gave her fresh frozen plasma to replete her antithrombin levels in addition to other plasmatic proteins. However, this did not result in any change in activated partial thromboplastin time, increasing the probability of an antithrombin-independent resistance to heparin. We did not retest the levels of serum antithrombin after infusion of antithrombin III and after the patient's clinical recovery, a limitation in confirming our hypothesis.
Another hypothesis leading to heparin resistance was that the patient's pro-inflammatory state and extracorporeal circulation were causing a non-antithrombin-mediated sequestration of heparin through increased heparin-binding proteins, as seen in patients with sepsis and in patients on extracorporeal circulatory support. ${ }^{4,5}$ In sepsis, activated neutrophils release various heparin-binding proteins, resulting in heparin resistance. ${ }^{4}$ Similar to sepsis, extracorporeal circulation also causes the release of heparin-binding proteins such as platelet factor 4 and interleukins, causing a decrease in the bioavailability of heparin. ${ }^{5}$

\section{Causes of thrombocytopenia}

Our patient's platelet count decreased substantially. A heparininduced thrombocytopenia assay was negative twice and we hypothesized that the thrombocytopenia was secondary to sepsis, contributing to either bone marrow suppression, or increased consumption or destruction of platelets. ${ }^{6}$ Along with thrombocytopenia, the patient's hemoglobin also decreased, raising concern for a consumptive coagulopathy. However, without any clinical evidence of disseminated intravascular coagulation, and with negative hemolysis markers, normal INR and aPTT and unremarkable blood film, this diagnosis was less likely. Similar to thrombocytopenia, bone marrow suppression most probably contributed to the decrease in synthesis of red blood cells. ${ }^{7}$

A potential explanation for the drop in platelet count may be related to a direct aggregating effect of heparin on platelets (i.e., a mild transient thrombocytopenia). If this had been the case, the platelet count would typically have fallen within the first 2 to 3 days after initiation of heparin and recovered over a similar time frame once heparin was stopped. However, the patient did not have a mild thrombocytopenia. She had a substantial decrease in platelet count from 111 to 13 from postadmission days 0 to 5 . The patient showed a drop in platelet count before commencing unfractionated heparin (on postadmission day 4), which increases the robustness of our hypothesis that her thrombocytopenia was induced by sepsis.

The patient had a low clinical probability of heparin-induced thrombocytopenia, according to the 4Ts score ${ }^{8}$ (thrombocytopenia, timing of platelet count fall, thrombosis or other sequelae, and other causes for thrombocytopenia present). Her total 4Ts score was 1 point, giving her a low probability $(<1 \%)$ risk of heparin-induced thrombocytopenia (Box 1$){ }^{8}$

We elected to perform ELISA (PF-4) assay to rule out heparin-induced thrombocytopenia, rather than other tests such as the serotonin-release assay. At our institution, we perform heparin-induced thrombocytopenia ELISA only. Patients are considered to have a positive result only if they present with an optical density of 2 or more. In addition, there is no indication for a serotonin-release assay, once an ELISA for heparin-induced thrombocytopenia is negative. To avoid false-positive serotoninrelease assays, an adjunct positive ELISA test is usually required. ${ }^{9}$ Thus, we perform serotonin-release assay only when results are equivocal (i.e., high clinical probability with ELISA of medium or low optical density). 
Box 1: Clinical probability of heparin-induced

thrombocytopenia using the 4Ts scoring system ${ }^{8}$ in a

41-year-old woman with heparin resistance on extracorporeal membrane oxygenation

\begin{tabular}{|c|c|c|}
\hline 4Ts scoring system criteria & Clinical course of patient & Points* \\
\hline Thrombocytopenia & $\begin{array}{l}\text { Platelet count fall, with } \\
\text { nadir between } 10 \text { and } \\
19 \times 10^{9} / \mathrm{L}\end{array}$ & 1 \\
\hline Timing of platelet count fall & $\begin{array}{l}4 \mathrm{~d} \text { (platelet count } 111 \text { on } \\
\text { postadmission day } 0 \text { and } \\
56 \text { on postadmission day } 4 \\
\text { with fluctuating levels on } \\
\text { postadmission day } 5 \text { from } \\
87 \text { to } 13 \text {; no previous } \\
\text { exposure to heparin }\end{array}$ & 0 \\
\hline $\begin{array}{l}\text { Thrombosis or other } \\
\text { sequelae }\end{array}$ & $\begin{array}{l}\text { Veno-venous extracorporeal } \\
\text { membrane oxygenation and } \\
\text { its anticoagulation started } \\
\text { on postadmission day 4; no } \\
\text { signs of thrombosis }\end{array}$ & 0 \\
\hline $\begin{array}{l}\text { Other causes for } \\
\text { thrombocytopenia present }\end{array}$ & None present & 0 \\
\hline
\end{tabular}

\section{Choice of anticoagulation}

Argatroban, a direct thrombin inhibitor, has been previously proposed as an alternative to heparin for the anticoagulation of patients on ECMO. ${ }^{10}$ Our patient had severe heparin resistance, making argatroban an adequate choice of anticoagulation. The veno-venous ECMO circuit that was used is heparin coated and would have had to be replaced by a non-heparin-coated circuit if the heparin-induced thrombocytopenia assay had been positive, to prevent thrombus formation and clotting of the ECMO filter. Given that both heparin-induced thrombocytopenia assays were negative, the heparin-coated circuit was maintained and its function was preserved under argatroban anticoagulation. Although argatroban was the agent used in this case, other direct thrombin inhibitors, such as bivalirudin, could have been used instead. ${ }^{11}$
A few days after we began argatroban, the patient's thrombocytopenia gradually resolved. Although we also stopped heparin at that time, giving the impression of a possible heparin-induced thrombocytopenia, both heparin-induced thrombocytopenia assays were negative and the patient did not present any signs of arterial or venous thrombosis. Therefore, we believe that the improvement in her thrombocytopenia was mostly owing to the amelioration of her sepsis and pro-inflammatory status.

\section{Conclusion}

This case discusses a severe presentation of pneumosepsis requiring the installation of a veno-venous ECMO circuit associated with acquired heparin resistance, and immune-mediated anemia and thrombocytopenia, without clinical or laboratory evidence of heparin-induced thrombocytopenia or consumptive coagulopathy. Given the limited literature on the prevalence of sepsis-induced heparin resistance in patients on extracorporeal membrane oxygenation, this case report adds a useful insight into such phenomenon.

\section{References}

1. Eikelboom JW, Hirsh J. Monitoring unfractionated heparin with the aPTT: time for a fresh look. Thromb Haemost 2006;96:547-52.

2. Maclean PS, Tait RC. Hereditary and acquired antithrombin deficiency. Drugs 2007;67:1429-40.

3. Marciniak E, Gockerman JP. Heparin-induced decrease in circulating antithrombin-III. Lancet 1977;2:581-4.

4. Linder A, Christensson $\mathrm{B}$, Herwald $\mathrm{H}$, et al. Heparin-binding protein: an early marker of circulatory failure in sepsis. Clin Infect Dis 2009;49:1044-50.

5. Finley A, Greenberg C. Heparin sensitivity and resistance: management during cardiopulmonary bypass. Anesth Analg 2013;116:1210-22.

6. Levi M. Platelets in sepsis. Hematology 2005;10(Suppl 1):129-31.

7. Piagnerelli M, Boudjeltia KZ, Gulbis B, et al. Anemia in sepsis: the importance of red blood cell membrane changes. Transfus Altern Transfus Med 2007;9:143-9. doi: 10.1111/j.1778-428X.2007.00072.x.

8. Cuker A, Gimotty PA, Crowther MA, et al. Predictive value of the 4Ts scoring system for heparin-induced thrombocytopenia: a systematic review and meta-analysis. Blood 2012;120:4160-7.

9. Warkentin TE, Arnold DM, Nazi I, et al. The platelet serotonin-release assay. Am J Hematol 2015;90:564-72.

10. Young G, Yonekawa KE, Nakagawa $\mathrm{P}$, et al. Argatroban as an alternative to heparin in extracorporeal membrane oxygenation circuits. Perfusion 2004;19:283-8.

11. Mulder MMG, Fawzy I, Lancé MD. ECMO and anticoagulation: a comprehensive review. Neth J Crit Care 2018;26:6-13.
Competing interests: Bob Kiaii reports receiving fees from Medtronic, Johnson and Johnson, and Boston Scientific, outside the submitted work. No other competing interests were declared.

This article has been peer reviewed.

The authors have obtained patient consent.
Affiliations: Division of Cardiac Surgery (Hage, Kiaii), Department of Surgery; Division of Hematology (Louzada), Department of Medicine, Western University, London Health Sciences Centre, London, Ont.

Contributors: All of the authors contributed to the conception and design of the work, and the acquisition, analysis, and interpretation of data. All of the authors drafted the manuscript, revised it critically for important intellectual content, gave final approval of the version to be published and agreed to be accountable for all aspects of the work.

Correspondence to: Bob Kiaii,

Bob.Kiaii@lhsc.on.ca 